Dear Author,

Please, note that changes made to the HTML content will be added to the article before publication, but are not reflected in this PDF.

Note also that this file should not be used for submitting corrections. 


\section{AUTHOR QUERY FORM}

\begin{tabular}{|l|l|l|}
\hline & Journal: JCLB & Please e-mail or fax your responses and any corrections to: \\
& Kumar, Anup \\
& & E-mail: Corrections.ESCH@elsevier.spitech.com \\
\hline ELSEVIER & Article Number: 3800 & Fax: +16196996721 \\
\hline
\end{tabular}

Dear Author,

Please check your proof carefully and mark all corrections at the appropriate place in the proof (e.g., by using on-screen annotation in the PDF file) or compile them in a separate list. Note: if you opt to annotate the file with software other than Adobe Reader then please also highlight the appropriate place in the PDF file. To ensure fast publication of your paper please return your corrections within 48 hours.

For correction or revision of any artwork, please consult http://www.elsevier.com/artworkinstructions.

We were unable to process your file(s) fully electronically and have proceeded by

Scanning (parts of) your article

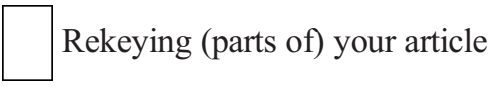
Scanning the artwork

Any queries or remarks that have arisen during the processing of your manuscript are listed below and highlighted by flags in the proof. Click on the 'Q' link to go to the location in the proof.

\begin{tabular}{|l|l|}
\hline Location in article & \multicolumn{1}{c|}{$\begin{array}{c}\text { Query / Remark: click on the Q link to go } \\
\text { Please insert your reply or correction at the corresponding line in the proof }\end{array}$} \\
\hline Q1 & Please confirm that given names and surnames have been identified correctly. \\
\hline Q2 & $\begin{array}{l}\text { Please provide an update for reference "Barnett et al., in press". } \\
\text { Please check this box if you have no } \\
\text { corrections to make to the PDF file. }\end{array}$ \\
\hline
\end{tabular}

Thank you for your assistance. 


\section{Clinical Biomechanics}

\section{Highlights}

\section{Longitudinal changes in transtibial amputee gait characteristics when negotiating a change in surface height during continuous gait}

C.T. Barnett ${ }^{\mathrm{a}, *}$, R.C.J. Polman ${ }^{\mathrm{b}, \mathrm{c}}$, N. Vanicek ${ }^{\mathrm{d}, \mathrm{e}}$

a SHAPE Research Group, School of Science and Technology, Nottingham Trent University, Nottingham, United Kingdom

${ }^{\mathrm{b}}$ Institute of Sport, Exercise and Active Living, Victoria University, Melbourne, Australia

c School of Public Health, Tropical Medicine and Rehabilitation Science, James Cook University, Cairns, Australia

d Department of Sport, Health and Exercise Science, University of Hull, United Kingdom

e Discipline of Exercise and Sport Science, Faculty of Health Sciences, University of Sydney, Australia

- Recent transtibial amputee stepping gait biomechanics were examined.

- The performance of stepping gait improved over time.

- Amputees adapted the lead limb preferences used during continuous stepping gait.

- Exploitation of intact limb function was crucial to stepping gait performance.

- Data provide an objective basis to inform therapeutic and prosthetic interventions. 


\title{
Longitudinal changes in transtibial amputee gait characteristics when negotiating a change in surface height during continuous gait
}

\author{
C.T. Barnett ${ }_{\Lambda}^{\mathrm{a}, *}, \mathrm{R}_{\Lambda}$.C.J. Polman ${ }_{\Lambda}^{\mathrm{b}, \mathrm{c}}, \mathrm{N}_{\Lambda}$. Vanicek ${ }_{\Lambda}^{\mathrm{d}, \mathrm{e}}$ \\ a SHAPE Research Group, School of Science and Technology, Nottingham Trent University, Nottingham, United Kingdom \\ ${ }^{\mathrm{b}}$ Institute of Sport, Exercise and Active Living, Victoria University, Melbourne, Australia \\ c School of Public Health, Tropical Medicine and Rehabilitation Science, James Cook University, Cairns, Australia \\ d Department of Sport, Health and Exercise Science, University of Hull, United Kingdom \\ e Discipline of Exercise and Sport Science, Faculty of Health Sciences, University of Sydney, Australia
}

\section{A R T I C L E I N F O}

\section{Article history:}

Received 26 November 2013

Accepted 8 May 2014

\section{Keywords:}

Gait

Longitudinal

Âmputee

Âctivity of daily living

Ŝtepping

R̂aised surface

K̂erb

\begin{abstract}
A B S T R A C T
Background: Negotiating a raised surface during continuous gait is an important activity of daily living and is a 21 potentially hazardous task with regards to trips, falls and fall-related injury. However, it is not known how recent 22 transtibial amputees adapt to performing stepping gait tasks in the 6-month period following discharge from 23 rehabilitation.

Methods: Recent transtibial amputees performed continuous gait trials, stepping onto and from a raised surface 25 walkway representing the height of a street kerb, whilst kinematic and kinetic data were recorded at one, 26 three and six months post-discharge from rehabilitation.

Findings: Walking speed increased when stepping down $(\mathrm{p}=0.04)$ and was invariant across the study period 28 when stepping up. At one month post-discharge, participants displayed an affected lead limb preference 29 (90.8\%) when stepping down and an intact lead limb preference (70.0\%) when stepping up, although 30 these lead limb preferences diminished over time. Participants spent more time in stance on the intact limb 31 compared to the affected limb in both stepping down (trail limb) $(p=0.01)$ and stepping up (lead and trail 32 limbs) $(p=0.05)$. Participants displayed significantly greater joint mobility and power bursts in the intact 33 trail limb when stepping down and in the intact lead limb when stepping up.

Interpretation: Transtibial amputees prefer to exploit intact limb function to a greater extent, although over time, 35 the means by which this occurs changes which affects the initial lead limb preferences. The results from the cur- 36 rent study enable future evidence-based therapeutic and prosthetic interventions to be designed that improve 37 transtibial amputee stepping gait.
\end{abstract}

(c) 2014 Published by Elsevier Ltd.

\section{Introduction}

The negotiation of a change in surface height during ongoing gait, such as stepping onto or from a pavement when crossing a road, is an important activity of daily living (ADL) that individuals are required to perform regularly (Begg and Sparrow, 2000; Buckley et al., 2008, 2011). When stepping down from a raised surface, the lead limb must control the downward momentum of the whole body centre of mass (COM) via eccentric muscle actions and conversely, when stepping up to a raised surface, it must perform positive work via concentric muscle actions, in order to raise the COM (Buckley et al., 2008, 2011; van Dieen et al., 2007, 2008). In both scenarios, the lead limb must be able to safely support bodyweight whilst providing propulsion in the context of ongoing gait and avoiding contact with the step.

\footnotetext{
* Corresponding author at: School of Science and Technology, Nottingham Trent University, Clifton Lane, Nottingham, United Kingdom, NG11 8NS.

E-mail address: cleveland.barnett@ntu.ac.uk (C.T. Barnett).
}

Although stepping gait may be executed by young able-bodied indi- 57 viduals without apparent difficulty (Barbieri et al., 2013; Begg and 58 Sparrow, 2000; Buckley et al., 2011; van Dieen et al., 2007, 2008), it is 59 more mechanically challenging compared to level gait (Nadeau et al., 60 2003). To the authors' knowledge, no data have been reported previous- 61 ly on the development of lower limb amputee (LLA) stepping gait. How- 62 ever, investigations into LLA function during challenging motor tasks 63 similar to stepping gait, such as stair negotiation and obstacle crossing, 64 have outlined specific biomechanical adaptations which may also be 65 adopted during LLA stepping gait. For example, during stair descent, 66 transtibial amputees (TTA) maintain the affected lead limb in an 67 extended position in an attempt to reduce the demands on the knee ex- 68 tensor musculature, avoiding potential limb buckling, whilst during 69 stair ascent intact trail limb ankle plantarflexion and knee extension 70 during stance aids the elevation of the COM in preparation for affected 71 limb stance (Aldridge et al., 2012; Alimusaj et al., 2009; Jones et al., 72 2006; Powers et al., 1997; Ramstrand and Nilsson, 2009; Schmalz 73 et al., 2007; Vanicek et al., 2010; Winter and Sienko, 1988). When 74 
negotiating obstacles, recent TTAs also display an inter-limb asymmetry in joint kinematics and kinetics, preferring to lead with the intact limb shortly following discharge from rehabilitation (Barnett et al., in press).

With these results in mind, it could be suggested that stepping gait may also present recent TTAs with a challenging task given that movement strategies are still being established. Subsequently, this may increase the potential for falling and fall-related injury, which are worldwide major public health concerns. Lower limb amputees have been shown to fall more frequently than age-matched controls (Miller et al., 2001), indicating that the impact of falls may be exacerbated in this population.

Previous research has documented significant long-term biomechanical adaptations in recent LLA level gait, obstacle negotiation, and balance activities, (Barnett et al., 2009, 2013a, 2013b, in press; Jones et al., 2001; Vrieling et al., 2009). Understanding how TTAs develop strategies for the successful completion of ADLs following formal rehabilitation is important as it establishes an objective evidence base from which further potential therapeutic or prosthetic interventions can be designed. Specifically, recent TTAs are likely to continue adapting their stepping gait strategies following discharge from rehabilitation. Therefore, understanding how this process occurs longitudinally with a view to optimising targeted clinical interventions is pertinent given that physical function in recent TTAs has been linked to quality of life and fear of falling (Barnett et al., 2013a, 2013b).

Therefore, the aim of the current study was to investigate biomechanical changes that occur when stepping onto and from a raised surface, in recent TTAs, during the six-month period following discharge from rehabilitation. Previous research has shown long-term adaptation to ADL during this time period following discharge from rehabilitation (Barnett et al., 2013a, 2013b, in press). It was predicted that walking speed would increase over time, reflecting an improvement in overall task performance. In addition, it was predicted that self-selected lead limb preference (LLP) would change over time reflecting changes in participants' preferred movement strategies, as previously reported in obstacle crossing (Barnett et al., in press). Finally, it was predicted that improvements in task completion and changes to LLP would be underpinned by increased intact limb joint mobility (peak joint angles and ranges of motion) and power bursts (peak joint powers), as seen previously during obstacle crossing (Barnett et al., in press).

\section{Methods}

\subsection{Participants}

Having completed rehabilitation within a national healthcare physiotherapy department, A consecutive sample of unilateral TTAs were recruited and gave informed consent to participate in the current 118 study. Participants were excluded if they experienced pain or discom- 119 fort whilst using their prostheses, had any current musculoskeletal inju- 120 ries or cognitive deficits. Participants were included if they were at least 121 18 years of age, were able to use their prosthesis to complete a number 122 of functional tasks without the use of a walking aid, including walking a 123 distance of five metres and stepping onto/from a pavement. The study 124 was approved by a local national healthcare service research ethics 125 committee (08/H1304/10).

\subsection{Experimental set-up}

In order to assess the biomechanical adaptations in stepping gait, a 128 custom raised-surface walkway ( $5 \mathrm{~m}$ length, $1.5 \mathrm{~m}$ width) was con- 129 structed with a step height that replicated a standard roadside kerb 130 $(7.5 \mathrm{~cm})$ and placed within a $10 \mathrm{~m}$ walkway (Buckley et al., 2005b, 131 2010; Jones et al., 2005, 2006) (Fig. 1). A ten-camera motion capture 132 system (Qualisys, Gothenburg, SE) and two force platforms (Kistler, 133 Model No: 9281B, Kistler, Winterthur, CH) sampled synchronous 134 kinematic $(100 \mathrm{~Hz})$ and ground reaction force (GRF) $(1000 \mathrm{~Hz})$ data via 135 Qualisys Track Manager software v2.8 (Qualisys, Gothenburg, SE). 136

\subsection{Experimental design and protocol}

137

A longitudinal repeated measures design was employed with partic- 138 ipants attending standardised data collection sessions at one, three and 139 six months following discharge from their rehabilitation programme. 140 Participants wore their own comfortable, flat footwear and were able 141 to fit and re-adjust their own prostheses prior to data collection. 142 Segmental six degree-of-freedom kinematics of the lower limbs were 143 recorded by attaching reflective markers $(14 \mathrm{~mm}$ ) bilaterally to the pos- 144 terior aspect of calcaneus, dorsum of the $2^{\text {nd }}$ metatarsal, medial and lat- 145 eral malleoli, medial and lateral femoral epicondyles, greater trochanter, 146 superior aspect of iliac crest, anterior-superior iliac spines, posterior- 147 superior iliac spines in accordance with the six degrees-of-freedom 148 marker set (Buczek et al., 2010; Cappozzo et al., 1995; Collins et al., 149 2009). Four-marker rigid clusters were securely attached to the thigh 150 and shank segments. Marker placement on the affected limb was esti- 151 mated from anatomical landmarks on the intact limb (Barnett et al., 152 2009; Powers et al., 1998). A static calibration was performed by 153 collecting kinematic data of each participant standing in the anatomical 154 neutral position. Following several practice trials to ascertain a self- 155 selected starting position, participants walked towards and stepped 156 onto the walkway, continued to walk, turned $180^{\circ}$ and then returned 157 along the walkway before stepping off, at a self-selected pace. This 158 allowed for the capture of continuous gait while stepping onto and 159
A

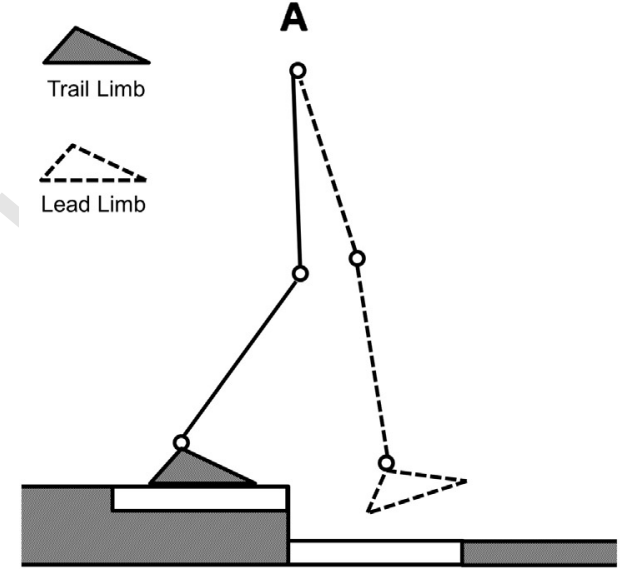

B

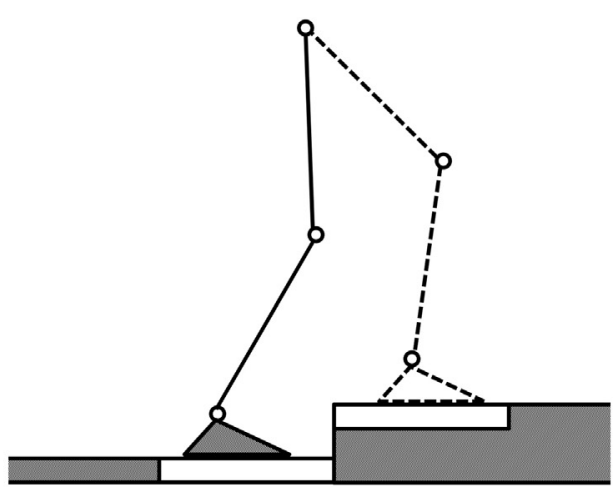

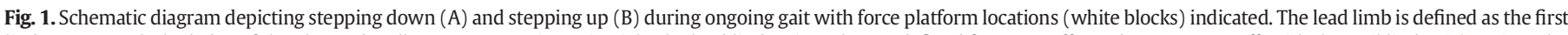

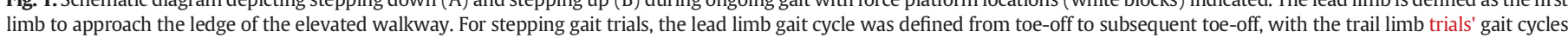
being defined from foot contact to subsequent foot contact. 
from a new level with a minimum of five and a maximum of ten trials being recorded for each task across multiple time periods.

\subsection{Data Analysis}

Raw kinematic and GRF data were exported to Visual 3D (C-Motion, Inc, Germantown, USA), interpolated using a cubic spline algorithm and filtered using a fourth-order low pass Butterworth filter with cut-off frequencies of $6 \mathrm{~Hz}$ and $30 \mathrm{~Hz}$ respectively. Medial and lateral landmarks defined anatomical frames from which segment co-ordinate systems were defined following the right hand rule (Cappozzo et al., 1995). An XYZ Cardan sequence was used to define the order of rotations to calculate joint kinematics. For stepping trials, data from the transition step, as participants stepped onto/from the raised-surface, were analysed (Fig. 1). The lead limb was defined as the first limb to approach/lead from the elevated walkway; the contralateral limb was designated as the trail limb. Self-selected LLPs were noted during the performance of each stepping trial using the motion capture video playback and calculated as percentages for both the intact and affected limbs (Fig. 1). Gait events were identified using GRF data in order to normalise data to the gait cycle as defined in Fig. 1.

Walking speed ( $\mathrm{m} \mathrm{s}^{-1}$ ) and stance duration (\% gait cycle) were calculated along with joint angle data for the ankle, knee and hip $\left({ }^{\circ}\right)$. Kinetic data were recorded following stepping for the lead limb and prior to stepping for the trail limb (Fig. 1). Peak ground reaction forces in the vertical (Fz) and anterior-posterior (Fy) directions were normalised to body weight (BW). Normalised peak joint power $(\mathrm{W} / \mathrm{kg})$ data were calculated for the ankle, knee and hip joints using standard inverse dynamics procedures.

In addition to the reporting of standard gait biomechanics data, task specific variables were selected based upon their relevance to the role of a particular limb during stepping gait (Barnett et al., in press). Therefore, during stepping down, lead limb variables that related to the controlled lowering of the COM during stance (e.g. load rate, peak joint angles during loading response and knee power burst K1) and to trail limb support of body weight during lead limb swing (e.g. joint ranges of motion (ROM) during single limb support and peak knee and hip power bursts $\mathrm{K} 1$ and $\mathrm{H} 2$ during mid-stance) were analysed. Similarly, during stepping up, lead limb variables that related to the raising and progression of the COM (e.g. peak joint power generation bursts throughout stance phase, A2, K2 and H3) were selected whilst variables related to trail limb progression and clearance were analysed (e.g. peak knee and hip flexion during swing).

\subsection{Statistical analysis}

Group mean data were analysed using a linear mixed model, Limb (Affected, Intact) ${ }^{*}$ Time (One, Three and Six Months) with repeated measures on the last factor allowing for analyses of changes in multiple gait variables (Brown and Prescott, 2006). Each feature of the design (Time and Limb) was modelled as a fixed effect with the appropriate covariance structure being selected according to the lowest value for Hurvich and Tsai's Criterion, indicating improved model fit (Bias Corrected Akaîke Information Criteria). Underlying assumptions were checked using conventional graphical methods and were deemed plausible unless stated otherwise. In the instance of a significant result, posthoc comparisons were conducted using a Sidak adjustment in IBM SPSS v19.0 (IBM, Portsmouth, UK). The alpha level of statistical significance was set at $\mathrm{p} \leq 0.05$.

\section{Results}

Participant details are presented in Table 1.

\subsection{Stepping down temporal-spatial}

217

Walking speed increased between one and six months post-discharge 218 $(\mathrm{p}=0.04)$ with both an affected (36\%) and intact (24\%) LLP (Table 2). 219 The affected LLP diminished between one month (90.8\%) and six months 220 (52.6\%) post-discharge (Table 2 ). Intact trail limb stance duration was 221 greater than affected trail limb stance duration $(\mathrm{p}=0.01)$ with trail 222 limb stance durations decreasing between one and three $(p=0.04) 223$ and one and six months $(\mathrm{p}=0.01)$ post-discharge (Table 2$)$, although 224 no significant interaction effect was present.

\subsection{Stepping down joint kinematics}

Lead limb peak ankle plantarflexion $(\mathrm{p}=0.01$ ) and peak knee 227 flexion ( $p=0.01$ ) during loading response were greater with an intact 228 LLP compared to an affected LLP (Fig. 2). Ankle ROM during stance 229 $(\mathrm{p}<0.01)$ and knee ROM during single limb support $(\mathrm{p}=0.05)$ were 230 both greater with an intact trail limb compared to an affected trail 231 limb (Fig. 2).

\subsection{Stepping down GRF and joint kinetics}

During early stance, intact limb load rate $(p=0.02)$, initial peak 234 vertical GRF (Fz1) $(\mathrm{p}=0.05)$ and peak posterior GRF (Fy1) $(\mathrm{p}<0.01) 235$ were significantly higher compared to the affected limb (Fig. 2). A sig- 236 nificant increase in lead limb peak anterior GRF (Fy2) ( $p=0.02)$ was 237 observed between one and six months post-discharge (Fig. 2). A signif- 238 icant interaction effect was reported for trail limb peak posterior (Fy1) 239 GRF ( $p=0.01)$ as this was generally greater in the intact limb 240 (Fig. 2). Peak anterior GRF (Fy2) ( $\mathrm{p}=0.01$ ) was significantly greater 241 with an intact trail limb compared to an affected trail limb (Fig. 2). 242

Peak lead limb knee power absorption during swing (K4) was 243 greater in the intact vs. affected limb ( $\mathrm{p}=0.01$ ) (Fig. 2). Peak ankle 244 power absorption (A1) $(\mathrm{p}=0.01)$ and generation $(\mathrm{A} 2)(\mathrm{p}=0.04) 245$ and peak knee power generation during stance $(K 2)(p=0.05)$ were 246 increased with an intact trail limb compared an affected trail limb 247 (Fig. 2). Peak knee power absorption during swing (K4) reduced over 248 time with an affected trail limb with variable changes in the intact 249 trail limb, resulting in a significant interaction effect $(p=0.03) 250$ (Fig. 2). Peak power absorption during stance (H2) increased signifi- 251 cantly between one and three months post-discharge $(\mathrm{p}=0.04)$. A sig- 252 nificant time main effect was also reported for peak hip power 253 absorption in pre-swing H3 ( $\mathrm{p}=0.05$ ), although post-hoc analysis 254 did not reveal the time points between which the significant increases 255 occurred.

\subsection{Stepping up temporal-spatial}

Walking speed was comparable at six months post-discharge 258 irrespective of LLP (Table 2). The predominately intact LLP at one 259 month post-discharge $(70.0 \%)$ decreased at six months post-discharge 260 (54.6\%) (Table 2). Intact limb stance duration was significantly greater 261 when acting as both the lead $(\mathrm{p}=0.02)$ and trail limb $(\mathrm{p}=0.05) 262$ (Table 2).

\subsection{Stepping up joint kinematics}

Lead limb ankle ROM during stance $(\mathrm{p}=0.02)$ and peak knee flex- 265 ion during loading response $(\mathrm{p}<0.01)$ were significantly greater with 266 an intact LLP compared to an affected LLP (Fig. 3). Peak plantarflexion 267 during swing was greater when trailing with the intact limb compared 268 to the affected $\operatorname{limb}(\mathrm{p}=0.01$ ). 
t1.1 Table 1

t1.2 Participant characteristics and prosthetic componentry of unilateral transtibial amputees.

t1.3 Gender (M/F) Age (years) $\quad$ Height $(\mathrm{m})$ Mass $(\mathrm{kg}) \quad$ Amputated limb (R/L) Cause of amputation Functional prosthetic components

\begin{tabular}{|c|c|c|c|c|c|c|c|}
\hline M & 44 & 1.77 & 76.5 & $\mathrm{R}$ & Non-vascular & Renegade freedom foot* & Socket interface devices, pylons and feet were \\
\hline t1.5 & 63 & 1.74 & 83.7 & $\mathrm{~L}$ & Non-vascular & Tres foot with torque absorber & consistent over time. All ankle feet complexes \\
\hline $\mathrm{t} 1.6$ & 44 & 1.82 & 81.0 & $\mathrm{R}$ & Non-vascular & Renegade freedom foot* & allowed for similar axial movement with the \\
\hline $\mathrm{t} 1.7$ & 75 & 1.93 & 101.9 & $\mathrm{~L}$ & Vascular & Multiflex ankle and foot & addition of specific differences highlighted. \\
\hline $\mathrm{t} 1.8$ & 50 & 1.83 & 106.6 & $\mathrm{R}$ & Vascular & Senator freedom foot ${ }^{*}$ & \\
\hline $\mathrm{t} 1.9$ & 41 & 1.92 & 95.4 & $\mathrm{R}$ & Vascular & Multiflex ankle and foot & \\
\hline $\mathrm{t} 1.10$ & 70 & 1.74 & 96.7 & $\mathrm{R}$ & Vascular & Multiflex ankle and foot & \\
\hline Mean (SD) & $56.1(14.9)$ & $1.82(0.08)$ & $91.7(11.4)$ & & & & \\
\hline
\end{tabular}

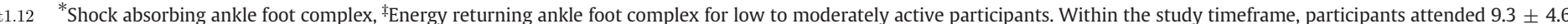

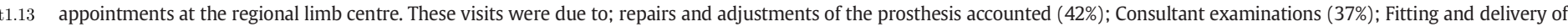
t1.14 a prosthetic component (18\%) and castings (3\%).

\subsection{Stepping up GRF and joint kinetics}

Intact lead limb peak posterior GRF (Fy1) was significantly greater when compared to the affected limb ( $p=0.01$ ) (Fig. 3). Both load rate and peak posterior GRF (Fy1) were greater with an intact trail limb vs. and affected trail limb at one month post-discharge and converged six months post-discharge, resulting in significant interaction effects ( $p=0.03$ and $p=0.05$, respectively) (Fig. 3 ).

Peak ankle power generation (A2) ( $p=0.02)$, peak knee power generation during stance $(\mathrm{K} 2)(\mathrm{p}<0.01)$ and peak knee power absorption during swing $(K 4)(p<0.01)$ were significantly greater with an intact LLP compared to an affected LLP (Fig. 3). Peak knee power absorption during late stance (K3) increased over time and was greater with an intact LLP resulting in a significant interaction effect $(\mathrm{p}=0.01)$ (Fig. 3).

Peak ankle power generation (A2) ( $p=0.02)$, peak knee power absorption during loading response $(K 1)(p=0.05)$ and peak knee power generation during stance $(K 2)(\mathrm{p}<0.01)$ were greater with an intact vs. affected trail limb (Fig. 3). An initial increase followed by a subsequent decrease in peak knee power absorption during late stance (K3) resulted in a significant time main effect between three and six months postdischarge $(\mathrm{p}=0.02)$ (Fig. 3).

\section{Discussion}

The current study investigated biomechanical changes that occur when stepping onto and from a raised surface, in recent TTAs during the six-month period following discharge from rehabilitation.

\subsection{Stepping down}

As predicted, there was an overall improvement in task performance as represented by a significant increase in walking speed. Participants initially preferred to lead with the affected limb, although at sixmonths post-discharge, this LLP had all but ceased.

As indicated previously, research has sought to explain LLA stair descent ability by describing the function of the affected limb (Aldridge et al., 2012; Alimusaj et al., 2009; Jones et al., 2006; Schmalz et al., 2007; van Dieen et al., 2007). However, the results from the current study suggest that the initial affected LLP was based upon participants' preference to exploit the capacity of the intact trail limb dûring stance.

Participants had greater stance duration, displayed greater ankle and knee mobility and ankle, knee and hip power absorption bursts during intact vs. affected trail limb stance. These results indicated that participants initially preferred to exploit the capabilities of the intact limb to safely control the lowering of the whole body COM during trail limb stance and potentially an initial cautionary approach to stepping down, which has been reported in perturbed stepping down in older adults (Buckley et al., 2005a, 2005b).
Another factor that may have contributed to the initial affected LLP 314 was the observation of a greater propulsive mechanism in the intact 315 trail limb, reflected by higher ankle and knee power generation bursts 316 (A2, K2 and K3) and propulsive GRFs (Fy2) in stance when compared 317 to the affected limb. These results suggested that participants preferred 318 to propel the intact limb forwards, while in single limb support on a rel- 319 atively 'rigid' affected lead limb. These results are unsurprising given 320 that for many TTAs, it is reasonable to assume that intact limb function 321 is more readily utilised thus likely to adopt a more dominant role 322 (Barnett et al., in press). In addition, the current participant group 323 were encouraged to lead with their 'weaker' limbs when descending 324 stairs and steps during rehabilitation, which is likely to have influenced 325 this LLP at one month post-discharge.

326

However, the reduction of the affected LLP at six months post- 327 discharge reflected the underlying shift in the strategies used by partic- 328 ipants during stepping down gait which occurred alongside improve- 329 ments in overall task performance, characterised by increased walking 330 speed. Results suggested that adaptations did occur in affected trail 331 limb function resulting in an improved controlled lowering mechanism 332 and, although these adaptations did not result in repeatedly significant 333 interaction effects, this may have reflected participants' increased confi- 334 dence in utilising this strategy. In addition, results from the current 335 study suggested that task performance at six months post-discharge 336 was also underpinned by the increased exploitation of intact limb vs. af- 337 fected limb capacity, which had not changed significantly over time. The 338 lack of dorsiflexion possible in the trail limb prosthetic ankle joint dur- 339 ing single limb support is likely to have necessitated the increased lead 340 limb intact ankle plantarflexion in late swing, as has been reported pre- 341 viously in LLA stair descent (Alimusaj et al., 2009; Schmalz et al., 2007). 342 This mechanism would have allowed participants to probe the ground 343 before 'falling' onto the intact lead limb in weight acceptance (Buckley 344 et al., 2008; Schmalz et al., 2007). In addition, it could be suggested 345 foot contact occurs earlier and more energy is absorbed by the lead 346 limb when utilising a toe first contact when stepping down onto the in- 347 tact limb, compared to a heel first contact, with a dorsiflexed prosthetic 348 ankle, with the affected limb (van Dieen et al., 2008). Furthermore, in- 349 creased intact lead limb loading during touchdown, as reflected by 350 GRF data, and greater observable but not statistically significant peak 351 joint powers bursts compared to the affected limb, suggested that the 352 intact lead limb knee extensor and ankle plantarflexor musculature 353 were more capable of lowering the body in a controlled fashion, corrob- 354 orating the mechanisms underpinning an intact LLP.

\subsection{Stepping up}

Overall task performance, as indicated by walking speed, was consis- 357 tent over time with an affected LLP and, although improvements in task 358 performance over time were noted with an intact LLP, these effects were 359 not statistically significant.

Initially, participants utilised an intact LLP strategy. However, while 361 stance duration did not change over time, it was greater in intact limb 362 
Table 2

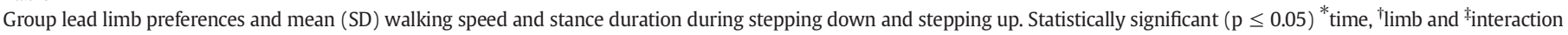
effects are highlighted.

\begin{tabular}{|c|c|c|c|c|c|}
\hline Task & Variable & Limb & One Month & Three Months & Six Months \\
\hline \multirow[t]{9}{*}{ Stepping Down } & \multirow[t]{3}{*}{ Lead limb preference (\%) } & Affected & 90.8 & 77.2 & 52.6 \\
\hline & & Intact & 9.2 & 22.8 & 47.4 \\
\hline & & Number of trials & $5.8(0.5)$ & $6.0(1.3)$ & $6.6(1.3)$ \\
\hline & \multirow[t]{2}{*}{ Walking speed $\left(\mathrm{m} \mathrm{s}^{-1}\right)$} & Lead affected* & $0.72(0.2)$ & $0.88(0.2)$ & $0.98(0.1)$ \\
\hline & & Lead intact* & $0.79(0.0)$ & $0.96(0.2)$ & $0.98(0.2)$ \\
\hline & \multirow[t]{4}{*}{ Stance duration (\% gait cycle) } & Lead affected & $58(4.1)$ & $58(2.7)$ & $57(2.0)$ \\
\hline & & Lead intact & $60(8.4)$ & $60(2.7)$ & $59(4.3)$ \\
\hline & & Trail affected ${ }^{*} \dagger$ & $71(1.3)$ & $66(2.1)$ & $66(2.0)$ \\
\hline & & Trail intact ${ }^{*} \dagger$ & $73(3.2)$ & $71(3.1)$ & $70(3.6)$ \\
\hline \multirow[t]{9}{*}{ Stepping Up } & \multirow[t]{3}{*}{ Lead limb preference (\%) } & Affected & 30.0 & 37.6 & 45.4 \\
\hline & & Intact & 70.0 & 62.4 & 54.6 \\
\hline & & Number of trials & $6.3(1.3)$ & $5.7(1.0)$ & $6.7(1.0)$ \\
\hline & \multirow[t]{2}{*}{ Walking speed $\left(\mathrm{m} \mathrm{s}^{-1}\right)$} & Lead affected & $0.94(0.0)$ & $1.01(0.1)$ & $0.94(0.1)$ \\
\hline & & Lead Intact & $0.76(0.1)$ & $0.92(0.1)$ & $0.93(0.2)$ \\
\hline & \multirow[t]{4}{*}{ Stance duration (\% gait cycle) } & Lead Affected $\dagger$ & $63(0.0)$ & $63(2.0)$ & $64(2.5)$ \\
\hline & & Lead Intact $†$ & $70(3.8)$ & $68(3.0)$ & $68(3.1)$ \\
\hline & & Trail Affected $\dagger$ & $62(2.4)$ & $60(1.8)$ & $59(2.8)$ \\
\hline & & Trail Intact $†$ & $63(0.0)$ & $63(1.7)$ & $64(3.8)$ \\
\hline
\end{tabular}

compared to the affected limb, regardless of role (lead or trail limb) which may have reflected a reluctance to transfer weight onto the affected limb (Powers et al., 1997). In the current study, an explanation for the initial intact LLP were related to the observations of greater intact 366 limb ankle and knee joint mobility demands and power bursts during 367 stance, as reflected by ankle and knee joint kinematic and peak joint 368
A

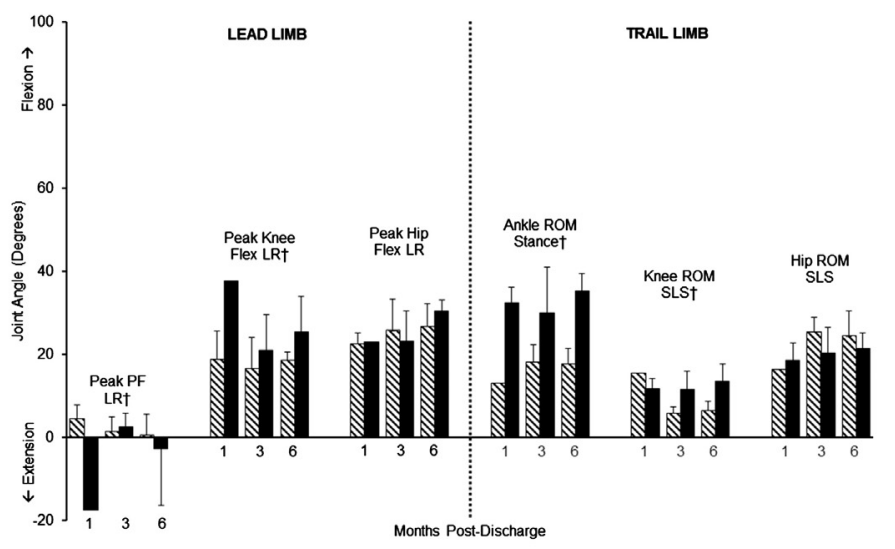

B

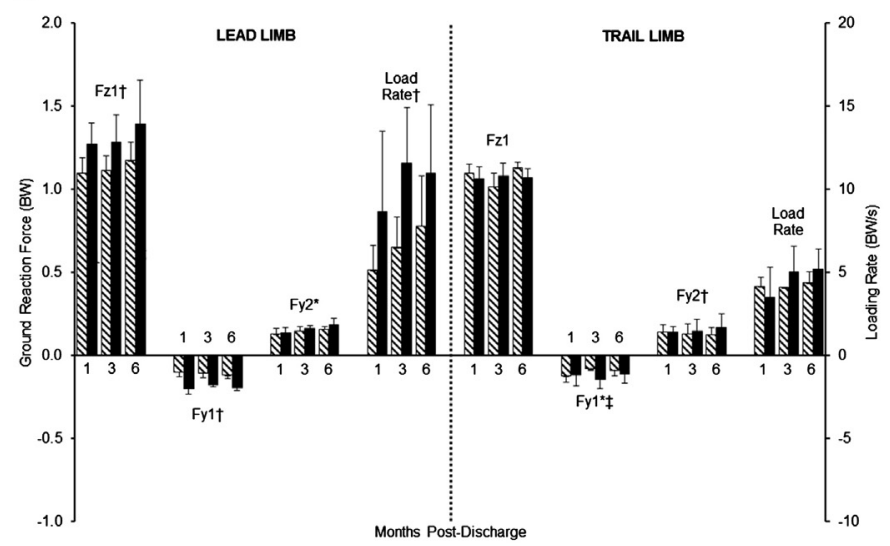

C

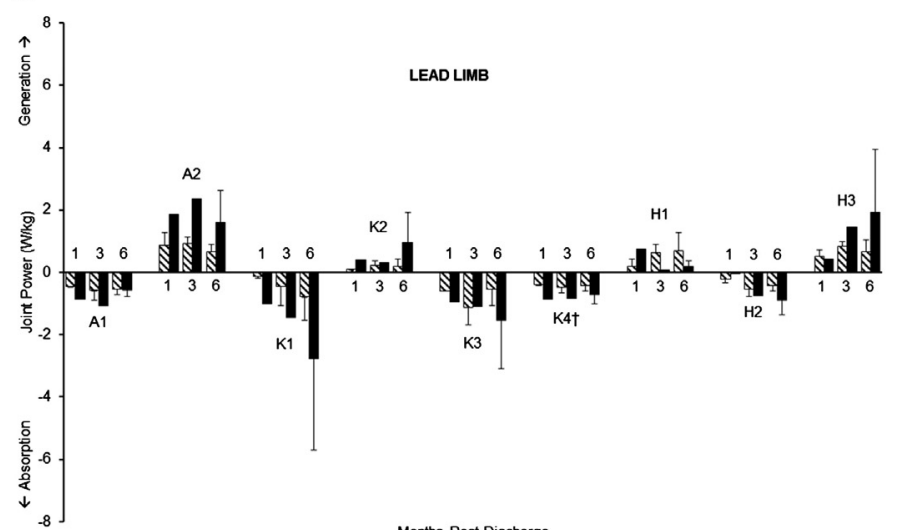

D

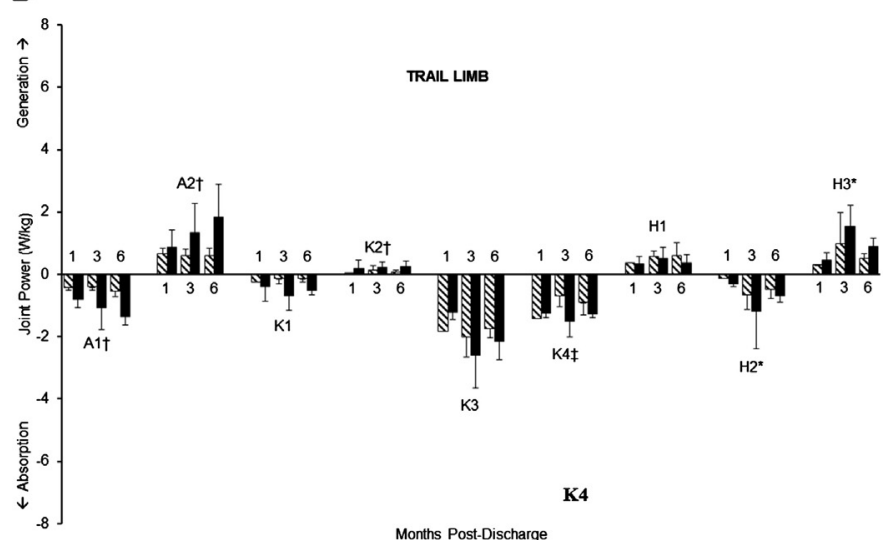

\section{$\mathbb{N}$ Affected Limb $\quad$ Intact Limb}

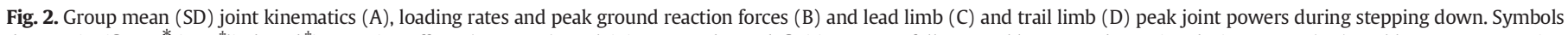

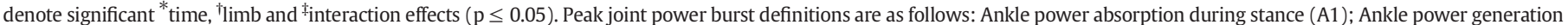

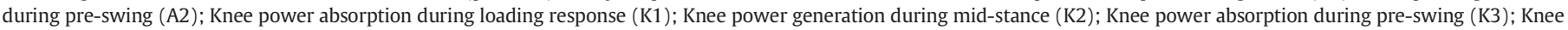

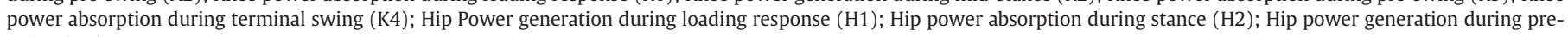
swing $(\mathrm{H} 3)$. 
A

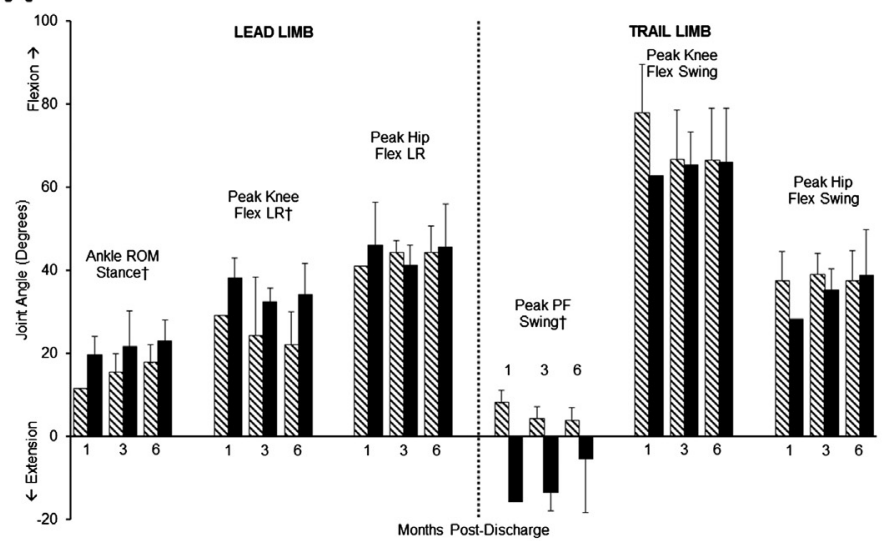

C

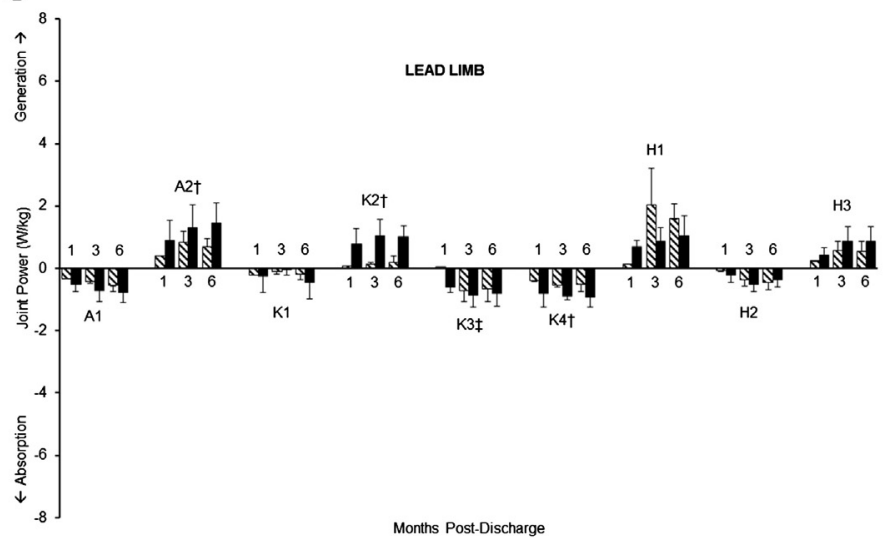

B

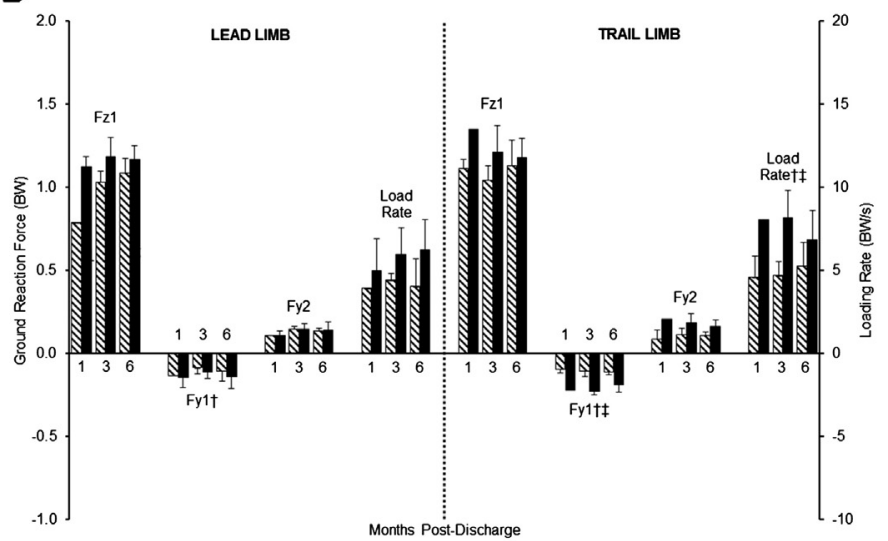

D

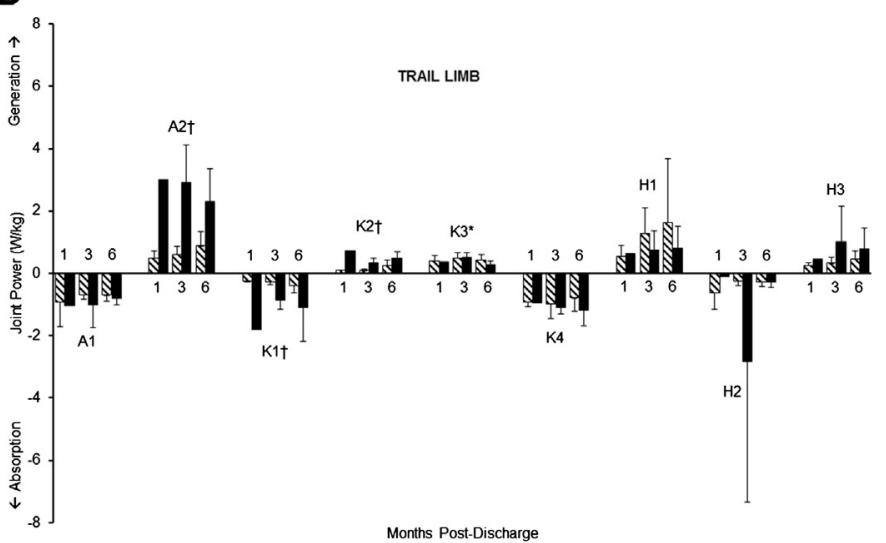

$\mathbb{A}$ Affected Limb $\quad$ Intact Limb

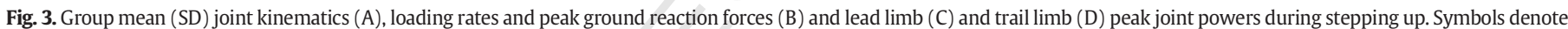

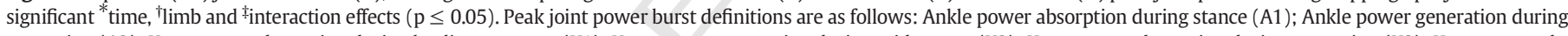

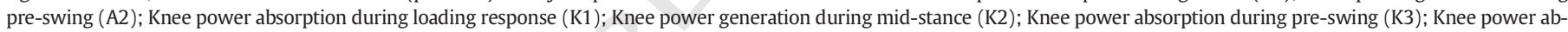
sorption during terminal swing (K4); Hip Power generation during loading response (H1); Hip power absorption during stance (H2); Hip power generation during pre-swing (H3).

power burst data, respectively. During stance, participants preferred to exploit the capacity of the intact lead limb in order to manage weight acceptance following foot contact and then do positive work in order to raise the COM and maintain progression in preparation for swing. Thus, as predicted, the higher utilisation of intact limb capacity initially led to its preferential use as the lead limb one month following discharge. It must also be stated that, conversely to stepping down gait, participants were encouraged to utilise an intact LLP during rehabilitation when stepping up stairs and steps. Therefore, it is probable that this effect persisted into the timeframe of the current study.

A shift from an initial intact LLP to more balanced LLP strategies at six months post-discharge occurred in stepping up gait, with comparable walking velocities observed throughout. This suggested that participants were more flexible in their strategy selection when performing the task. Participants spent more time in intact trail limb stance with an affected LLP and during this period, the intact limb experienced greater loading, as reflected by increased GRFs. In addition, increased peak joint power generation and absorption bursts were associated with the intact limb indicating that it aided the control of whole body momentum in preparation for stepping up during early stance with continued progression prior to swing. These results corroborated previous research highlighting the role of the intact trail limb in the elevation of the COM in more experienced LLAs (Schmalz et al., 2007). Seemingly, the participants in the current study who adapted to using the affected limb as their lead limb, increased their flexibility of strategy selection.
While these individuals may have been better equipped to deal with un- 394 predictable configurations of the physical environment, these adapta- 395 tions in strategy selection occurred despite a persistent disparity 396 between the capacity of the intact vs affected limbs.

\subsubsection{Summary}

To the authors' knowledge, the current longitudinal study is the first 399 to investigate the biomechanical changes present in the stepping gait of 400 recent TTAs. Following discharge from rehabilitation, participants' over- 401 all performance of stepping down from and stepping up to a raised sur- 402 face displayed trends towards improvement. Moreover, participants' 403 willingness to deviate from an initial preferred strategy could be 404 interpreted as a positive increase in plasticity when completing this 405 motor task. Participants preference to exploit intact limb function may 406 be beneficial initially, although potential problems may arise in the fu- 407 ture when a situation does not allow for the self-selection of a particular 408 LLP and thus, necessitates a strategy requiring increased utilisation of af- 409 fected limb function. An example of such a situation would be the pre- 410 sentation of an unexpected change in surface height where it could be 411 assumed TTA stepping performance would be reduced or even become 412 hazardous given that TTAs have been shown to perform worse under in- 413 creasing time pressure during an obstacle avoidance task (Hofstad et al., 414 2006). Therefore, it is important that TTAs are adaptable in terms of LLP 415 selection and do so according to the task requirements rather than a 416 preference to utilise the capacity of a particular limb. Results from the 417 
current study have implications for TTAs rehabilitation as they suggest further functional utilisation of the affected limb is required, as the disparity in utilisation was evident at one month and persisted at sixmonths post-discharge. Interventions aimed at encouraging the use and exploration of different strategies in safe, controlled but challenging environments may address this disparity. In addition, interventions targeting the eccentric lowering mechanism and concentric raising mechanism of the knee extensors within the affected limb would benefit stepping down and stepping up gait respectively, particularly in the early stages following discharge. Such training may in turn reduce TTAs falls risk by increasing adaptability when performing stepping gait. It is possible that these changes may be achieved through affected limb resistance and flexibility training aimed at improving knee extensor strength and joint mobility. Also, the prescription of advanced prosthetic components and improved prosthetic design aimed at increasing ankle mobility may also aid TTAs functional performance, thus investigation into the effects of these interventions are warranted.

\subsubsection{Limitations}

Although the results from the current study were obtained over a six-month period in recent TTAs, it is not possible to elucidate what the long-term health effects are arising from the apparent adaptations in stepping performance. Research has shown that asymmetries in LLA mechanics may be linked to bone health, although further causal relationships must be established (Sherk et al., 2008). Given the small sample size of this study, variation in participants' cause of amputation may have limited statistical power. The assessment of one step height representing a street kerb may not have induced the biomechanical adaptations associated with a more challenging step height. Finally, variation in prosthetic componentry may have increased the variation in some biomechanical variables reported.

\section{Conclusion}

Following discharge from rehabilitation, trends towards improvement in task performance occur in stepping gait. Although LLPs changed over time, reflecting an increased flexibility in strategy selection, TTAs continued to exploit intact limb function to a greater extent when compared to the affected limb, regardless of the role being performed. The novel data presented provide an objective basis on which an understanding of how TTAs learn to perform this important ADL can be structured, thus informing future therapeutic and prosthetic interventions.

\section{Declaration of Interest}

The authors report no conflicts of interest. The authors alone are responsible for the content and writing of the paper.

\section{Acknowledgements}

This project was financially supported by the Owen Shaw Award from the Circulation Foundation, UK. The Circulation Foundation had no involvement in the design, execution or reporting of the study. Authors would like to thank Mrs Amanda Hancock, Mrs Barbara Brown and Mrs Lynne Smith for their help during patient recruitment.

\section{References}

Aldridge, J.M., Sturdy, J.T., Wilken, J.M., 2012. Stair ascent kinematics and kinetics with a powered lower leg system following transtibial amputation. Gait Posture 36, 291-295. Alimusaj, M., Fradet, L., Braatz, F., Gerner, H.J., Wolf, S.I., 2009. Kinematics and kinetics with an adaptive ankle foot system during stair ambulation of transtibial amputees. Gait Posture 30, 356-363.
Barbieri, F.A., Lee, Y., Gobbi, LT.B., Pijnappels, M. Van Dieen, JH. 2013. The effect of mus- 472 cle fatigue on the last stride before stepping down a curb. Gait Posture 37, 542-546. 473 Barnett, C., Vanicek, N., Polman, R., Hancock, A., Brown, B., Smith, L., Chetter, I., 2009. Ki- 474 nematic gait adaptations in unilateral transtibial amputees during rehabilitation. 475 Prosthetics Orthot. Int. 33, 135-147.

Barnett, C.T., Vanicek, N., Polman, R.C.J., 2013a. Temporal adaptations in generic and 477 population-specific quality of life and falls efficacy in men with recent lower-limb 478 amputations. J. Rehabil. Res. Dev. 50, 437-448.

Barnett, C.T., Vanicek, N., Polman, R.C.J., 2013b. Postural responses during volitional and 480 perturbed dynamic balance tasks in new lower limb amputees: A longitudinal 481 study. Gait Posture 37, 319-325.

Barnett, C.T., Polman, R.C., Vanicek, N., 2014. Longitudinal kinematic and kinetic adapta- Q2 tions to obstacle crossing in recent lower limb amputees. Prosthetics Orthot. Int. 484 (in press).

Begg, R.K., Sparrow, W.A., 2000. Gait characteristics of young and older individuals nego- 486 tiating a raised surface: Implications for the prevention of falls. J. Gerontol. Ser. A Biol. 487 Sci. Med. Sci. 55, 147-154.

Brown, H., Prescott, R., 2006. Applied mixed models in medicine, 2nd ed. Wiley, 489 Chichester,

Buckley, J.G., Heasley, K., Scally, A., Elliott, D.B., 2005a. The effects of blurring vision on 491 medio-lateral balance during stepping up or down to a new level in the elderly. 492 Gait Posture 22, 146-153.

Buckley, J.G., Heasley, K.J., Twigg, P., Elliott, D.B., 2005b. The effects of blurred vision on the 494 mechanics of landing during stepping down by the elderly. Gait Posture 21, 65-71. 495

Buckley, J.G., MacLellan, M.J., Tucker, M.W., Scally, A.J., Bennett, S.J., 2008. Visual guidance 496 of landing behaviour when stepping down to a new level. Exp. Brain Res. 184, 497 223-232.

Buckley, J.G., Jones, S.F., Johnson, L., 2010. Age-differences in the free vertical moment 499 during step descent Clin. Biomech. 25, 147-153.

Buckley, J.G., Timmis, M.A., Scally, A.J., Elliott, D.B., 2011. When Is Visual Information Used 501 to Control Locomotion When Descending a Kerb? PLoS ONE 6, e19079.

Buczek, F.L., Rainbow, M.J., Cooney, K.M., Walker, M.R., Sanders, J.O., 2010. Implications of 503 using hierarchical and six degree-of-freedom models for normal gait analyses. Gait 504 Posture 31, 57-63.

Cappozzo, A., Catani, F., Della Croce, U., Leardini, A., 1995. Position and orientation in 506 space of bones during movement: anatomical frame definition and determination. 507 Clin. Biomech. 10, 171-178.

Collins, T.D., Ghoussayni, S.N., Ewins, D.J., Kent, J.A., 2009. A six degrees-of-freedom mark- 509 er set for gait analysis: Repeatability and comparison with a modified Helen Hayes 510 set. Gait Posture 30,173-180.

Hofstad, C.J., van der Linde, H., Nienhuis, B., Weerdesteyn, V., Duysens, J., Geurts, A.C., 512 2006. High failure rates when avoiding obstacles during treadmill walking in patients 513 with a transtibial amputation. Arch. Phys. Med. Rehabil. 87, 1115-1122.

Jones, M.E., Bashford, G.M., Bliokas, V.V., 2001. Weight-bearing, pain and walking velocity 515 during primary transtibial amputee rehabilitation. Clin. Rehabil. 15, 172-176. 516

Jones, S.F., Twigg, P.C., Scally, A.J., Buckley, J.G., 2005. The gait initiation process in unilat- 517 eral lower-limb amputees when stepping up and stepping down to a new level. Clin. 518 Biomech. 20, 405-413.

Jones, S.F., Twigg, P.C., Scally, A.J., Buckley, J.G., 2006. The mechanics of landing when 520 stepping down in unilateral lower-limb amputees. Clin. Biomech. 21, 184-193. 521

Miller, W.C., Speechley, M., Deathe, B., 2001. The prevalence and risk factors of falling and 522 fear of falling among lower extremity amputees. Arch. Phys. Med. Rehabil. 82, 523 1031-1037.

Nadeau, S., McFadyen, B.J., Malouin, F., 2003. Frontal and sagittal plane analyses of the 525 stair climbing task in healthy adults aged over 40 years: what are the challenges 526 compared to level walking? Clin. Biomech. 18, 950-959.

Powers, C.M., Boyd, L.A., Torburn, L., Perry, J., 1997. Stair ambulation in persons with 528 transtibial amputation: An analysis of the Seattle LightFoot(TM). J. Rehabil. Res. 529 Dev. 34, 9-18.

Powers, C.M., Rao, S., Perry, J., 1998. Knee kinetics in trans-tibial amputee gait. Gait Pos- 531 ture $8,1-7$.

Ramstrand, N., Nilsson, K., 2009. A comparison of foot placement strategies of transtibial 533 amputees and able-bodied subjects during stair ambulation. Prosthetics Orthot. Int. 534 33, 348-355.

Schmalz, T., Blumentritt, S., Marx, B., 2007. Biomechanical analysis of stair ambulation in 536 lower limb amputees. Gait Posture 25, 267-278.

Sherk, V.D., Bemben, M.G., Bemben, D.A., 2008. BMD and bone geometry in transtibial and 538 transfemoral amputees. J. Bone Miner. Res. 23, 1449-1457.

van Dieen, J.H., Spanjaard, M., Konemann, R., Bron, L., Pijnappels, M., 2007. Balance control 540 in stepping down expected and unexpected level changes. J. Biomech. 40, 541 3641-3649.

van Dieen, J.H., Spanjaard, M., Konemann, R., Bron, L., Pijnappels, M., 2008. Mechanics of 543 toe and heel landing in stepping down in ongoing gait. J. Biomech. 41, 2417-2421. 544

Vanicek, N., Strike, S.C., McNaughton, L., Polman, R., 2010. Lower limb kinematic and 545 kinetic differences between transtibial amputee fallers and non-fallers. Prosthetics 546 Orthot. Int. 34, 399-410.

Vrieling, A.H., van Keeken, H.G., Schoppen, T., Hof, A.L., Otten, B., Halbertsma, J.P.K., 548 Postema, K., 2009. Gait adjustments in obstacle crossing, gait initiation and gait ter- 549 mination after a recent lower limb amputation. Clin. Rehabil. 23, 659.

Winter, D.A., Sienko, S.E., 1988. Biomechanics of Below-Knee Amputee Gait. J. Biomech. 551 $21,361-367$ 